\title{
Buffer Size Optimization for Full-Search Block Matching Algorithms*
}

\author{
Yuan-Hau Yeh Chen-Yi Lee \\ Dept. of Electronics Engineering \& Institute of Electronics \\ National Chiao-Tung University, Hsin-chu 300, Taiwan, ROC \\ Tel: 886-3-5712121 Ext. 54238; E-mail: cylee@ royals.ee.nctu.edu.tw
}

\begin{abstract}
This paper presents how to find optimized buffer size for VLSI architectures of full-search block matching algorithms. Starting from the DG (dependency graph) analysis, we focus in the problem of reducing the internal buffer size under minimal $1 / O$ bandwidth constraint. As a result, a systematic design procedure for buffer optimization is derived to reduce realization cost.
\end{abstract}

\section{1: Introduction}

Block matching algorithms (BMA) are often found in image and video applications for pattern analysis, motion detection, data compression, etc. Inherent computational complexity in these algorithms often demand special hardware to meet real-time performance. Due to algorithm regularity and modularity, such algorithms are very suitable for VLSI implementation. However for practical system design considerations, not only computational requirements have to be coped with, but also memory bandwidth has to be minimized to reduce $\mathrm{I} / \mathrm{O}$ pin-count and hence realization cost. In other words, the desired hardware has to provide sufficient computational power to meet algorithm complexity. Moreover the large volumes of image/video data have to be managed carefully to enhance on-chip data reusability. From these viewpoints, a good VLSI architecture for BMA is often judged by the following issues: (1) internal storage space which determines on-chip memory cost and bandwidth, (2) $1 / O$ pin-count which determines $1 / O$ bandwidth and packaging cost, and (3) processor element ( $P E)$ efficiency which determines $P E$ structure and its utilization ratio for a given period.

Motion estimation (ME) based on full-search BMA (FBMA), one of the key techniques in video coding to remove redundancy, is a good example for illustration of such design complexity. Many research results on VLSI architectures for FSBMA were proposed in the past $[1,2,3,4,5,6,7,8,10]$, where research efforts have moved from meeting computational requirements to reducing memory bandwidth as well as providing multi-functions specified in standard [14]. Yeo and $\mathrm{Hu}[9]$ proved when the search range $(\mathrm{P})$ is set to half of the macroblock size $(\mathrm{N})$, significant reduction in $\mathrm{J} / \mathrm{O}$ bandwidth can be achieved without sacrificing performance. But in the case of $\mathrm{N}=\mathrm{P}$, four cascaded chips are needed, increasing the implementation cost of large search area. In the situation of large search area, data reusable problem becomes an important issue. In order to reduce $\mathrm{V} / \mathrm{O}$ bandwidth, some internal buffer is needed to greatly reduce $\mathrm{I} / \mathrm{O}$ bandwidth. Our goal targets at finding optimized buffer size for sufficient large search area ME architecture under minimum $/ \mathrm{O}$ bandwidth constraint in a single chip.

* Work supported by the National Science Council of Taiwan, R.O.C. under Grant NSC86-2221-E-009-016. 


\section{2: Algorithm fundamentals}

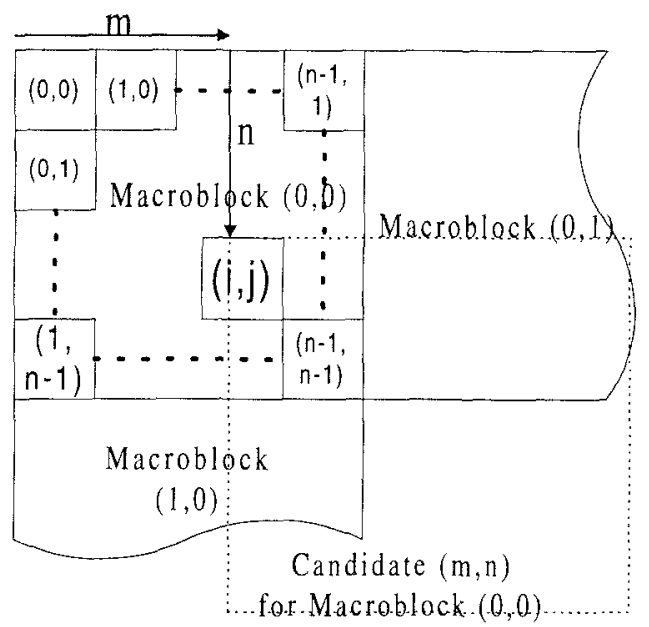

Figure 1: Image frame structure.

Usually, a picture frame is divided into many macroblocks, each macroblock contains $\mathrm{N}^{2}$ pixels, as shown in Figure 1. Assuming $N_{h}$ is the frame width and $N_{v}$ is the frame height, a frame is arranged as $N_{5} / h \times \mathrm{N} / \mathrm{r}$ macroblocks, $(h, v)$ are the horizontal and vertical macroblock indexes in one frame. For one macroblock, there are $(2 \mathrm{P})^{2}$ candidates inside its search area, $(m, n)$ are the horizontal and vertical candidate indexes for one macroblock. Looking inside the macroblock, $(i, j)$ are the horizontal and vertical pixels indexes in one macroblock. The matching criterion of the FBMA can be expressed as:

$$
\operatorname{MAD}(m, n)=\sum_{i=0}^{N-1} \sum_{j=0}^{N-1}|R(i, j)-S(i+m, j+n)|
$$

$R$ is called as "reference block" and $S$ is named as "search area". According to the formulas derived in [9], which are given below, a three-level for-loop algorithm can be formulated as shown in Figure 2(a).

$$
\left\{\begin{array}{l}
b=v \cdot N_{h}+h \\
0 \leq b<N_{h} \cdot N_{v} \\
h=b \bmod N_{h} \\
v=\left\lfloor\frac{b}{N_{h}}\right\rfloor
\end{array}\right.
$$$$
\left\{\begin{array}{l}
l=2 P(m+P)+n+P \\
0 \leq 1<(2 P)^{2} \\
m=1 \bmod 2 P-P \\
n=\left\lfloor\frac{1}{2 P}\right\rfloor-P
\end{array}\right.
$$$$
\left\{\begin{array}{l}
k=j \cdot N+j \\
0 \leq k<N^{2} \\
i=k \bmod N \\
j=\left\lfloor\frac{k}{N}\right\rfloor
\end{array}\right.
$$

If we swap the direction of $(m, n)$ and $(i, j)$, that is, the scan order of parameters $l$ and $k$ are changed, then we can get four basic types of DG. Figure 2(b) shows the search areas of two adjacent macroblocks $(\mathrm{N}=\mathrm{P}=2)$, the dark region indicates the common data of two search areas, and is one of the key points to reduce $\mathrm{V} / \mathrm{O}$ bandwidth. The four basic types of DG for two adjacent macroblocks are derived in Figure 3. 


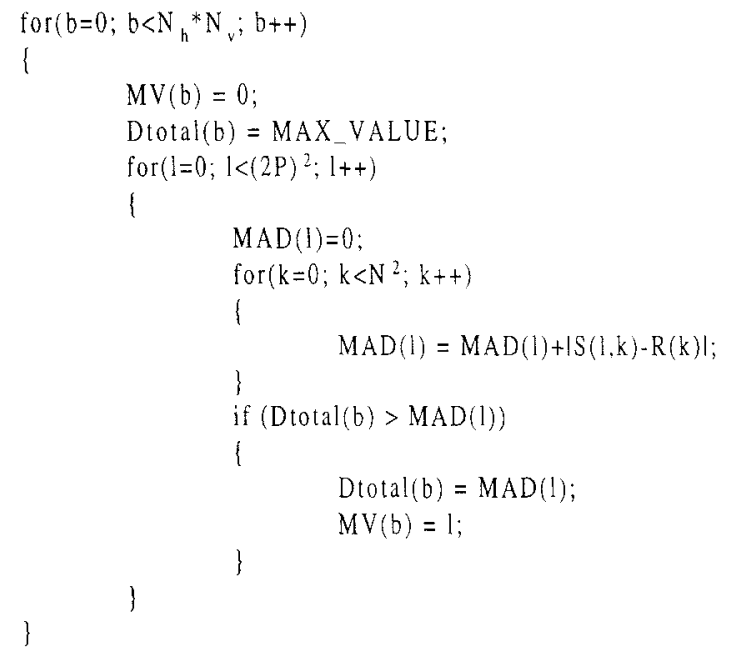

Figure 2: (a)Three-level loop algorithm for FBMA. (b) Search area of two adjacent

Figure 2: (a)Three-level loop algorithm for FBMA.
macroblocks.

\begin{tabular}{|l|l|l|l|l|l|l|}
\hline$S 11$ & $S 21$ & $S 31$ & $S 41$ & $S 51$ & $S 61$ & $S 71$ \\
\hline$S 12$ & $S 22$ & $S 32$ & $S 42$ & $S 52$ & $S 62$ & $S 72$ \\
\hline$S 13$ & $S 23$ & $S 33$ & $S 43$ & $S 53$ & $S 63$ & $S 73$ \\
\hline$S 14$ & $S 24$ & $S 34$ & $S 44$ & $S 54$ & $S 64$ & $S 74$ \\
\hline$S 15$ & $S 25$ & $S 35$ & $S 45$ & $S 55$ & $S 65$ & $S 75$ \\
\hline
\end{tabular}

Common Area of two search areas

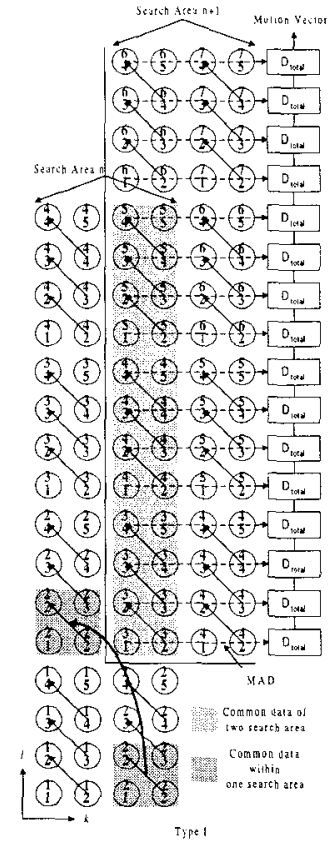

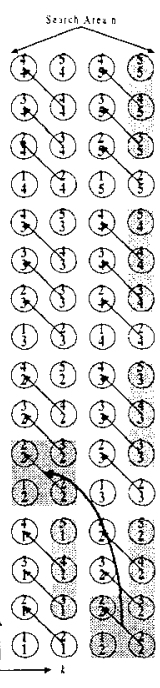
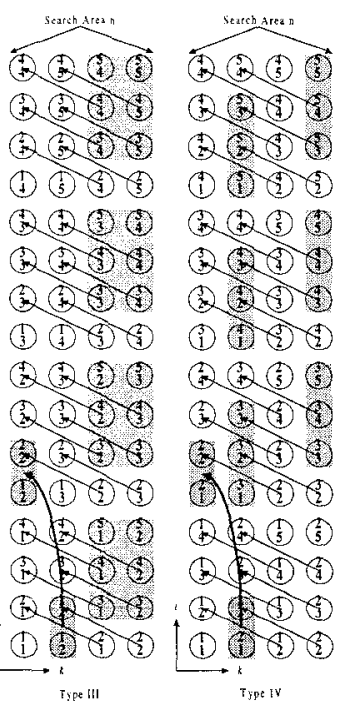

Figure 3. Four basic types of DG, from type I type IV.

Analyzing the DGs, we can extract the dependency arc $\vec{b}$ of all variables. Table 1 shows the dependency arcs which are necessary to evaluate buffer size for a given mapping strategy. $R$ is the reference block data, $D_{\text {total }}$ is the distortion value of a candidate block, $M V$ is the motion vector of one macroblock, $\mathrm{S}$ is the search area data. Data dependency in the search area are more complicate than other variables. In Figure 4, observing the DG of type I, there are three types of relation in $\mathrm{S}$-variables which will be discussed in detail in next section. From this table 
we know for different mapping directions, we can choose freely various dependency arcs unless they violate the mapping constraints. With this table, we can evaluate optimized memory size for different mapping directions under $\mathrm{V} / \mathrm{O}$ bandwidth constraint. Here we withdraw type III and type IV from our discussion because they cannot be used to generate cost-effective architecture mappings.

Table 1: Dependency arcs.

\begin{tabular}{|c|c|c|c|c|}
\hline & Type I & Type II & Type III & Type IV \\
\hline $\mathrm{R}, \mathrm{D}_{\text {total }}, \mathrm{MV}$ & {$[1,0]$} & {$[1,0]$} & {$[1,0]$} & {$[1,0]$} \\
\hline MAD & {$[0,1]$} & {$[0,1]$} & {$[0,1]$} & {$[0,1]$} \\
\hline $\mathrm{S}_{\text {within_block }}(\mathrm{S} 1)$ & {$[1,-1]$ or $[-1,1]$} & {$[1,-1]$ or $[-1,1]$} & {$[1,-\mathrm{N}]$ or $[-\mathrm{N}, 1]$} & {$[1,-\mathrm{N}]$ or $[-\mathrm{N}, 1]$} \\
\hline $\mathrm{S}_{\text {within_SA }}{ }^{*}(\mathrm{~S} 2)$ & {$[2 \mathrm{P},-\mathrm{N}]$} & {$[2 \mathrm{P},-\mathrm{N}]$} & {$[2 \mathrm{P},-1]$} & {$[2 \mathrm{P},-1]$} \\
\hline $\mathrm{S}_{\text {between SAA }}{ }^{* *}(\mathrm{~S} 3)$ & $\begin{array}{c}{[-2 \mathrm{P},-\mathrm{N}(\mathrm{N}-1)]} \\
\text { or }[-2 \mathrm{NP}, 0]\end{array}$ & {$[-1,-(\mathrm{N}-1)]$} & {$[-1,-\mathrm{N}]$} & {$[-2 \mathrm{P},-1]$} \\
\hline
\end{tabular}

* data dependency in the same search area.

** data dependency between two adjacent search areas.

Dependency arcs can be mapped to PE array and a set of dependency edges and delays are acquired in the following equation described in [12]:

$$
\left[\begin{array}{c}
D(\vec{e}) \\
\vec{e}
\end{array}\right]=\left[\begin{array}{c}
\vec{S}^{r} \\
P^{T}
\end{array}\right] \cdot[\vec{b}]
$$

where $D(\vec{e})$ is the delays on edge $\vec{e}$ in the PE array, $\vec{S}$ is the schedule vector, and $P$ is the processor basis. According to the delays of each dependency edge $\vec{e}$, we can derive total memory size for a giving mapping scheme.

\section{3: Buffer size estimation}

Memory elements can be classified as registers in each PE and memory banks outside the PE array as well.

Table 2: Delays on dependence edges.

\begin{tabular}{|c|c|c|c|c|}
\hline & Type I (a) & Type I (b) & Type II (a) & Type II (b) \\
\hline$\vec{d}$ & {$[1,0]$} & {$[0,1]$} & {$[1,0]$} & {$[0,1]$} \\
\hline$\vec{S}$ & {$[1,1]$} & {$[1,1]$} & {$[1,1]$} & {$[1,1]$} \\
\hline$P$ & {$[0,1]$} & {$[1,0]$} & {$[0,1]$} & {$[1,0]$} \\
\hline$D\left(\overrightarrow{e_{R}}\right)$ & 1 & 1 & 1 & 1 \\
\hline$D\left(\overrightarrow{e_{u A D}}\right)$ & 1 & 1 & 1 & 1 \\
\hline$D\left(\overrightarrow{e_{S 1}}\right)$ & 0 & 0 & 0 & 0 \\
\hline$D\left(\overrightarrow{e_{S 2}}\right)$ & $2 \mathrm{P}-\mathrm{N}$ & $2 \mathrm{P}-\mathrm{N}$ & $2 \mathrm{P}-\mathrm{N}$ & $2 \mathrm{P}-\mathrm{N}$ \\
\hline$D\left(\overrightarrow{e_{S 3}}\right)$ & $-2 \mathrm{NP}+(2 \mathrm{P})^{2} *$ & $\mathrm{~N}-2 \mathrm{P} * *$ & $(2 \mathrm{P})^{2}-\mathrm{N}$ & $\mathrm{N}^{2}-\mathrm{N}$ \\
\hline $\mathrm{PE}-$ count & $\mathrm{N}^{2}$ & $(2 \mathrm{P})^{2}$ & $\mathrm{~N}^{2}$ & $(2 \mathrm{P})^{2}$ \\
\hline
\end{tabular}

* $\quad(2 \mathrm{P})^{2}$ term indicates the effective latency of one search area.

** particular negative delay value will be described lately.

In Table 2 we know for above four conditions, $D\left(\overrightarrow{e_{R}}\right), D\left(\overrightarrow{e_{D_{t o x i l}}}\right)$ and $D\left(\overrightarrow{e_{M A D}}\right)$ are all equal to 1. 
These cause one or zero delay in each PE depending on the projection direction $\vec{d}$. For example, in type I(a), $D\left(\overrightarrow{e_{D_{\text {tonal }}}}\right)$ doesn't result in any delay in each $\mathrm{PE}$, while $D\left(\overrightarrow{e_{R}}\right)$ causes one delay in each PE. Above delays are inevitable, so the buffer minimization problem falls on exploiting both $D\left(\overrightarrow{e_{52}}\right)$ and $D\left(\overrightarrow{e_{53}}\right)$. In the following sub-sections we describe this problem of the four conditions respectively, where the condition $\mathrm{N}=\mathrm{P}$ is emphasized.

\section{1: Type I(a)}

First, from Table 2 we know $\overrightarrow{D\left(\vec{e}_{32}\right)}=2 \mathrm{P}-\mathrm{N}$ and $\overrightarrow{e_{S 2}}=-\mathrm{N}$. We can arrange $\mathrm{N}^{2}$ PEs as snake-like as shown in Figure 4. Applying the mapping procedure based on Equation 3, $\mathrm{N}^{*}(2 \mathrm{P}-\mathrm{N})$ registers are needed between two adjacent rows. But there exists data dependency among the data in $\mathrm{N}^{*}(2 \mathrm{P}-\mathrm{N})$ registers as illustrated in Figure 3.

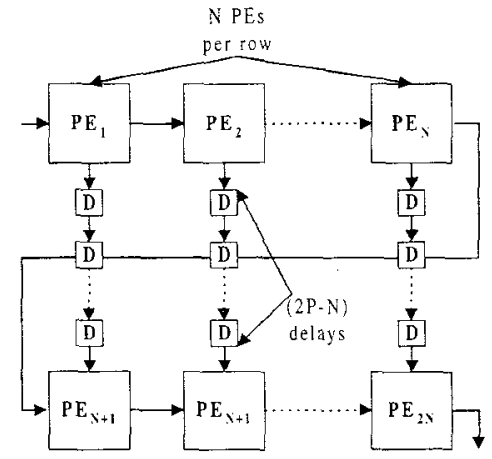

Figure 4: Delay elements for dependency edge $\vec{e}$.

Then we apply a procedure called "merging" in order to greatly reduce the register count. Before presenting this procedure, we first define the "boundary" and "non-boundary" regions in one search area as shown in Fig. 5(a). The sizes of these two regions are $(\mathrm{N}-1)^{*}(\mathrm{~N}+2 \mathrm{P}-1)$ and $(2 \mathrm{P}) *(\mathrm{~N}+2 \mathrm{P}-1)$ respectively. From the DG we know sometimes "non-boundary" and "boundary" data must be passed to the same row, like $(\mathrm{S} 21, \mathrm{~S} 15)$ or $(\mathrm{S} 31, \mathrm{~S} 25)$. If we assume $\mathrm{N}=\mathrm{P}$, two inequations are acquired:

$$
\begin{aligned}
& \text { delay }=2 P-N>N-1 \\
& \text { delay }=2 P-N<2 P
\end{aligned}
$$

$(2 \mathrm{P}-\mathrm{N})>(\mathrm{N}-1)$ means only $(\mathrm{N}-1)$ registers are sufficient to buffer "boundary" data and $(2 \mathrm{P}-$ $\mathrm{N})<(2 \mathrm{P})$ means at least $2 \mathrm{P}-\mathrm{N}$ (this value is right equal to $D\left(\overrightarrow{e_{s 2}}\right)$ which is the lower bound size to buffer "non-boundary" data) registers are needed to buffer "non-boundary" data. After applying "merging" procedure, we can reduce the buffer size from $\mathrm{N}^{*}(2 \mathrm{P}-\mathrm{N})$ to $(\mathbf{2 P}-\mathbf{1})$, as shown in Figure 5(b). Next, another key point to reduce the I/O bandwidth is to consider dependency arc S3 which represents the common data of two adjacent search areas as depicted in Figure 3. Figure 6 shows three conditions for different S3. One idea to reduce the buffer size is: the reusable data is passed to the buffer for finding next motion vector as later as possible. So, condition III is a good choice, then we get $\mathrm{S} 3=[-2 \mathrm{NP}, 0]$ and $D\left(\overrightarrow{e_{53}}\right)=2 \mathrm{P} *(2 \mathrm{P}-\mathrm{N})$. Observing the condition III in Figure 6 , from $(t+1)$ to $(t+2 P)$ in time axis, $(N+2 P-1)$ registers are needed to buffer the data. So, for $D\left(\overrightarrow{e_{S 3}}\right)=-2 \mathrm{NP}+(2 \mathrm{P})^{2}$, dependency edge $\overrightarrow{e_{S 3}}$ results in $(2 \mathrm{P}-\mathrm{N}) *(\mathrm{~N}+2 \mathrm{P}-1)$ 
buffer size.

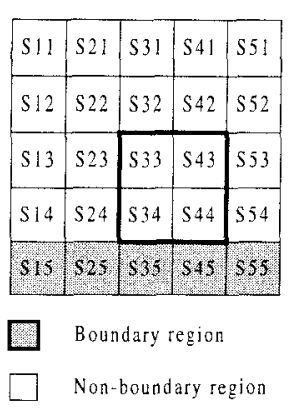

(a)

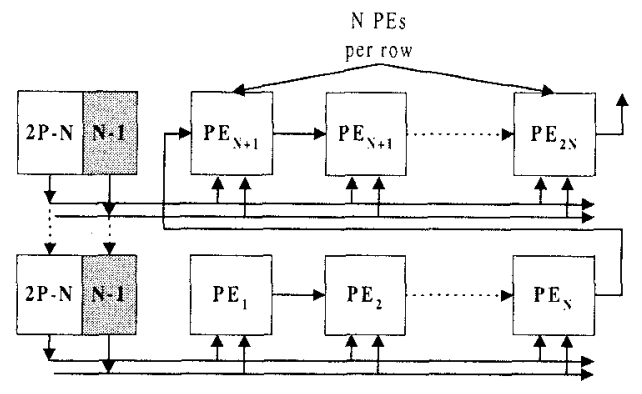

(b)

Figure 5: (a) "non-boundary" and "boundary" regions of one search area. (b) Buffer size after "merging" procedure.

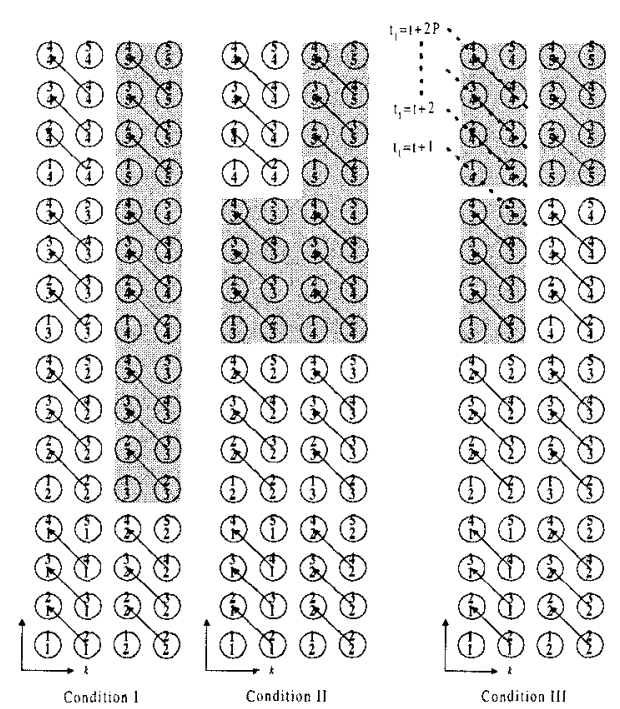

Figure 6: Three conditions for dependency arc S3.

According to above discussion, a formula for the buffer size estimation of DG type I(a) is given as follows:

$$
N^{2} \cdot\left(\vec{S} \cdot \vec{b}_{R}+\stackrel{-T}{s} \cdot \vec{b}_{M A D}+\vec{s}^{T} \cdot \vec{b}_{S 1}\right)+(N-1) \cdot(2 P-1)+(2 P-N) \cdot(N+2 P-1)
$$

\section{2: Type $\mathbf{I}(\mathbf{b})$}

This case causes $D\left(\overrightarrow{e_{s}}\right)=\mathrm{N}-2 \mathrm{P}<0$. It implies that $D\left(\overrightarrow{e_{S 3}}\right)$ violates the constraint $\vec{S}^{T} \cdot \vec{b} \geq 0$, however it is a special case for $\mathrm{N}=\mathrm{P}$ as shown in Fig. 7, where two adjacent search areas are appended together. 


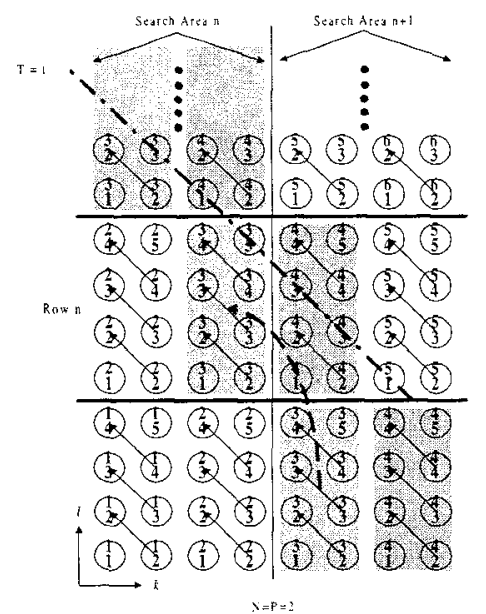

Figure 7: Data dependency between two search areas.

$D\left(\overrightarrow{e_{S 3}}\right)$ doesn't cost any buffer to hold data. Let's just consider $\overrightarrow{e_{S 2}} \cdot D\left(\overrightarrow{e_{S 2}}\right)=2 \mathrm{P}-\mathrm{N}$ and $\overrightarrow{e_{S 2}}=2 \mathrm{P}$. The $(2 \mathrm{P})^{2} \mathrm{PEs}$ can be arranged as snake-like form as shown in Fig. 8. At time $t$, three pixels (S35, S43 and S51) are sent to row n concurrently. After the "merging" procedure, we can reduce the buffer size from $2 \mathrm{P}^{*}(2 \mathrm{P}-\mathrm{N})$ to $(4 \mathrm{P}-\mathrm{N}-1)$ per row. The total buffer is giving as:

$$
(2 P)^{2} \cdot\left(\vec{s} \cdot \vec{b}_{R}+\vec{s}^{T} \cdot \vec{b}_{D_{\alpha b}}+\vec{s}^{T} \cdot \vec{b}_{M A D}+\vec{s}^{T} \cdot \vec{b}_{S I}\right)+(2 P-1) \cdot(4 P-N-1)
$$

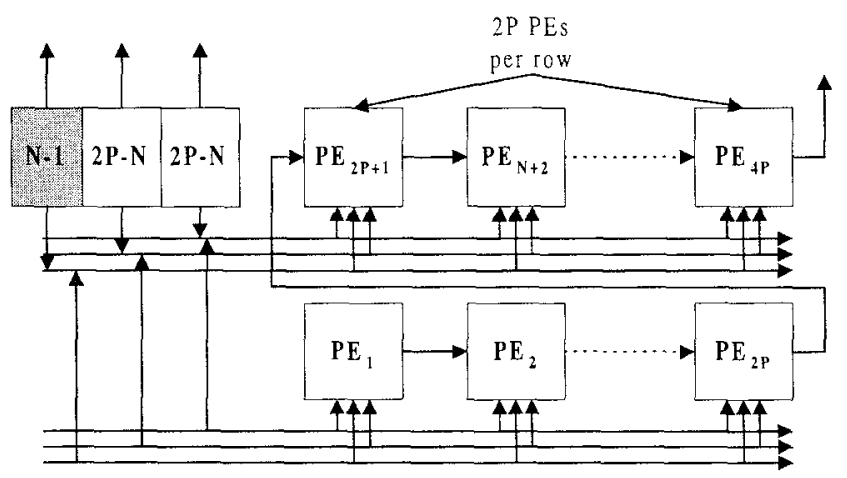

Figure 8: PE array and buffer structure of DG type $\mathbf{I}(\mathbf{b})$.

\section{3: Type II(a)}

In this case, the buffer size for $\overrightarrow{e_{S 2}}$ is the same as DG type I(a). From Table 2 we know $D\left(\overrightarrow{e_{S 3}}\right)=(2 \mathrm{P})^{2}-\mathrm{N}, \overrightarrow{e_{S 3}}=-(\mathrm{N}-1)$. Applying the concept described in section 3.1 , the whole common data between two adjacent search areas should be buffered, so the buffer size for $\overrightarrow{e_{S 3}}$ is $(2 \mathrm{P}-1) *(\mathrm{~N}+2 \mathrm{P}-1)$. The total buffer for $\mathrm{DG}$ type $\mathrm{\Pi}(\mathrm{a})$ is:

$$
N^{2} \cdot\left(\vec{S}^{T} \cdot \vec{b}_{R}+\vec{S}^{T} \cdot \vec{b}_{M A D}+\vec{S}^{T} \cdot \vec{b}_{S}\right)+(N-1)^{*}(N+P-1)+(2 P-1) *(N+2 P-1)
$$




\section{4: Type II(b)}

In this case, the buffer size for $\overrightarrow{e_{S 2}}$ is the same as DG type I(b) described in section 3.2.

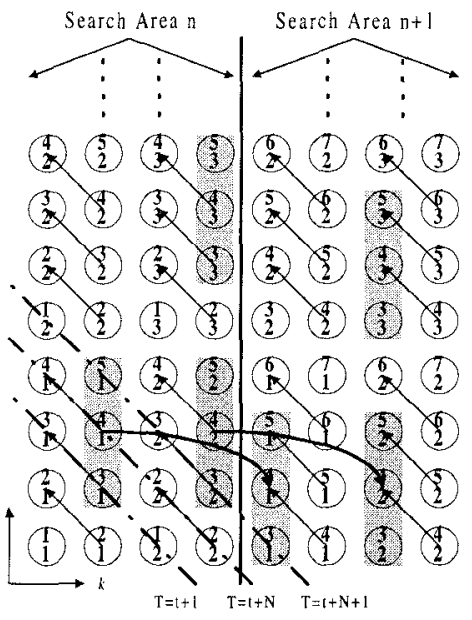

Figure 9: DG for type II(b).

Considering the illustrated example shown in Figure 9. At time $t+1$ to $t+N+1$, the common data (i.e. S31, S41 and S51, the size is 2P-1) between two adjacent search areas should be stored in the buffer every $\mathrm{N}$ cycles. For $D\left(\overrightarrow{e_{33}}\right)=\mathrm{N}^{2}-\mathrm{N}$, the buffer size for $\overrightarrow{e_{S 3}}$ is $\left(N^{2}-N\right) / N *(2 P-1)=(N-1) *(2 P-1)$. So, the total buffer size is given as:

$$
\left.(2 P)^{2} \cdot \stackrel{-T}{S} \cdot \vec{b}_{R}+\stackrel{-T}{S} \cdot \vec{b}_{D_{k t}}+\stackrel{+T}{S} \cdot \vec{b}_{M D}+\stackrel{+T}{S} \cdot \vec{b}_{\mathrm{S}}\right)+(2 P-1) \cdot(N+2 P-1)+(N-1) \cdot(2 P-1)
$$

\section{4: Comparison and mapped $\mathrm{ME}$ architectures}

The comparison of the proposed buffer size estimation with other existing papers is presented in Table 3

Table 3: Performance comparison based on $N=P=16, S_{h}=720, S_{v}=480, F_{r}=30$.

\begin{tabular}{|c|c|c|c|}
\hline Design issues & $\begin{array}{c}\text { Internal buffer size } \\
\text { (bytes) }\end{array}$ & $\begin{array}{c}\text { I/O bandwidth } \\
\text { (Mbits/sec) }\end{array}$ & $\begin{array}{c}\text { PE-count \& cycles } \\
\text { per MV }\end{array}$ \\
\hline Hsieh et. al[5] & 1489 & 99.8 & 256,2209 \\
\hline Baek et. al[6] & 1730 & 120 & 256,1760 \\
\hline Wang et. al[7] & 7616 & 52.2 & 1024,256 \\
\hline Yeo and Hu*[9] & 3072 & 93.3 & 1024,256 \\
\hline \hline Type I(a) & $\mathbf{1 7 2 9}$ & $\mathbf{4 2 . 1}$ & $\mathbf{2 5 6 , 1 0 2 4}$ \\
\hline Type I(b) & $\mathbf{4 5 2 9}$ & $\mathbf{4 2 . 1}$ & $\mathbf{1 0 2 4 , 2 5 6}$ \\
\hline Type II(a) & $\mathbf{2 4 3 4}$ & $\mathbf{4 2 . 1}$ & $\mathbf{2 5 6 , 1 0 2 4}$ \\
\hline Type II(b) & $\mathbf{4 9 9 4}$ & $\mathbf{4 2 . 1}$ & $\mathbf{1 0 2 4 , 2 5 6}$ \\
\hline
\end{tabular}

* Although this architecture costs small buffer size than Type I(a) and Type II(b), more VO bandwidth and pin-count (80 VS 24) are needed. 
In the following we present two ME architectures for type I(a) and type I(b) respectively.

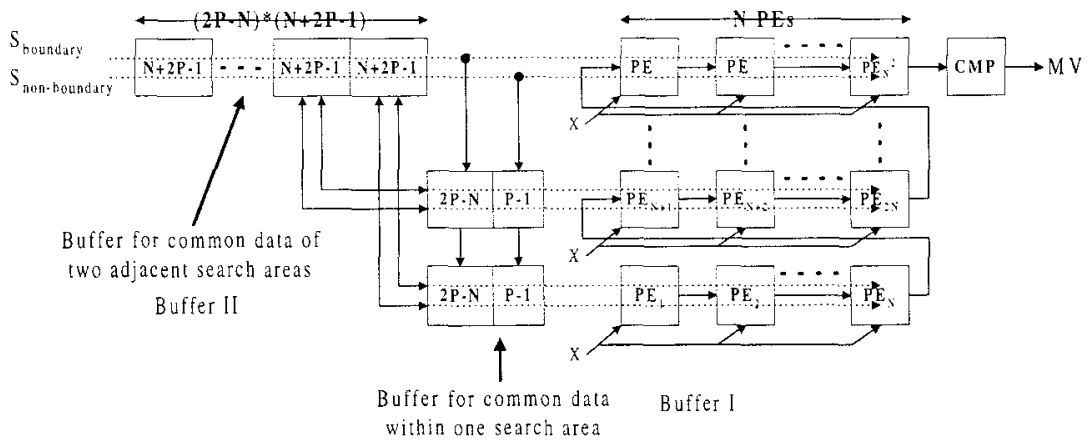

Figure 10: ME architecture for type I(a).

According to the discussion in section 3.1, beside registers in each PE, two extra buffers are used to buffer the data for $\overrightarrow{e_{S 2}}$ and $\overrightarrow{e_{S 3}}$. These two buffers are illustrated in Figure 10 as buffer I and buffer II respectively. The buffers can be implemented as pointer address memory described in our previous work[12]. There are two busses passing each row of the PE array, supplying "boundary" and "non-boundary" data concurrently. In order to reduce I/O bandwidth, the penalty paid is complex routing and the data broadcasting problem during changing to next search area .

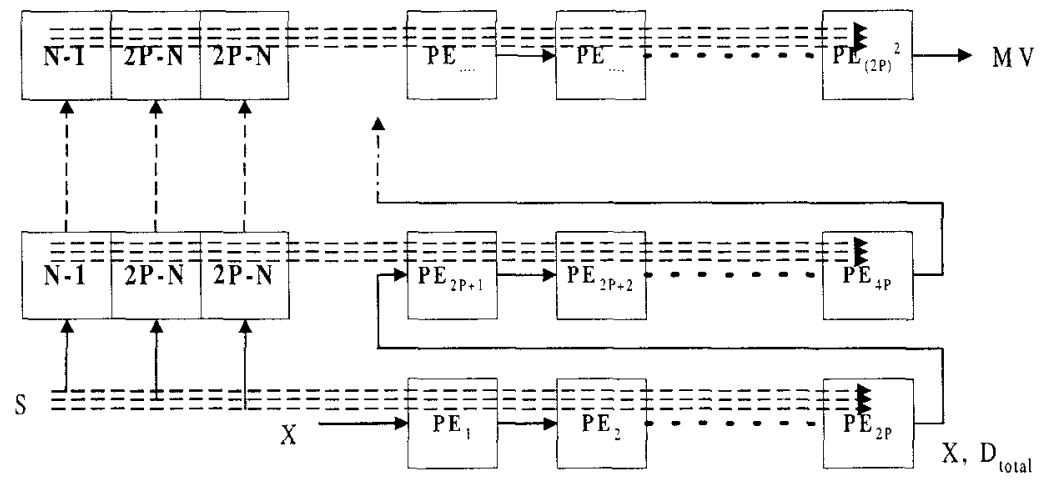

Figure 11: ME architecture for type $\mathbf{I}(\mathbf{b})$.

In Figure 11, the size of PE array is $(2 \mathrm{P})^{2}$. There are three busses passing each row of the PE array. The reason lies in that three pixels should be consumed at the same time in worst case.

\section{5: Conclusion}

In this paper we have presented how to find optimized buffer size derived from DG under minimal I/O bandwidth constraint. Optimized buffer size helps greatly reducing $\mathrm{I} / \mathrm{O}$ bandwidth with little penalty of implementation cost in ASIC design, especially in the condition of large search range. We have derived four basic types of DG and discussed the buffer size estimation corresponding to various mapping strategies. The buffer can be easily implemented by pointer address memory (PAM). Finally, two ME architectures with 100\% PE efficiency have also been demonstrated to show the proposed mapping schemes. 


\section{References}

[1] K K. Komarek and P. Pirsch, "Array Architectures for Block Matching Algorithms", IEEE Trans. on Circuits and Systems, Vol. 36, No. 10, Oct. 1989, pp. 1301-1308.

[2] R.C. Kim and S.U. Lee, "A VLSI Architecture for a Pel Recursive Motion Estimation Algorithm", IEEE Trans. on Circuits and Systems, Vol. 36, No. 10, Oct. 1989, pp. 1291-1300.

[3] L. De Vos and M. Stegherr, "Parameterizable VLSI Architectures for the Full-Search Block Matching Algorithm", IEEE Trans. on Circuits and Systems, Vol. 36, No. 10, Oct. 1989, pp. 1309-1316.

[4] K.M. Yang, M.T. Sun, and L. Wu, "A Family of VLSI Designs for the Motion Compensated Block-Matching Algorithm", IEEE Trans. on Circuits and Systems, Vol. 36, No. 10, Oct. 1989, pp. 1317-1325.

[5] C.H. Hsieh and T.P. Lin, "VLSI Architecture for Block-Matching Motion Estimation Algorithm", IEEE Trans. on Circuits and Systems for Video Technology, Vol. 2, No. 2, June 1992, pp. 169-175.

[6] J. Baek, S. Nam, M. Lee, C. Oh, and K. Hwang, "A Fast Array Architecture for Block Matching Algorithm", in Proc. of ISCAS'94, London, May 30-June 2, 1994, pp. 4.211-4.214.

[7] C.L. Wang, K.M. Chen, and J.M. Hsiung, "A High-Throughput and Flexible VLSI Architecture for Motion Estimation", in Proc. of ICASSP'95, Detroit, May 8-12, 1995, pp. 3295-3298.

[8] S. Chang, J.H. Hwang, and C.W. Jen, "Scalable Array Architecture Design for Full Search Block Matching", IEEE Trans. on CAS for Video Technology, Vol, 5, No. 4, Aug. 1995, pp. 332-343.

[9] H. Yeo and Y.H. Hu, "A Novel Modular Systolic Array Architecture for Full-Search Block Matching Motion Estimation", IEEE Trans, on CAS for Video Technology, Vol. 5, No. 5, Oct. 1995, pp. 407 - 416.

[10] L. De Vos and M. Schobinger, "VLSI Architecture for a Flexible Block Matching Processor", IEEE Trans. on CAS for Video Technology, Vol. 5, No. 5, Oct. 1995, pp. $417-428$

[11] H. T. Kung, "Why Systolic architectures", IEEE Computer, Vol. 15, no 1, Jan., 1982.

[12] S.Y. Kung, "VLSI Array Processors", Prentice-Hall International Editions, 1988

[13] G.L. Tzeng and C.Y. Lee, "An Efficient Memory Architecture for Motion Estimation Processor Design", in Proc. of ISCAS'95, Seattle, April 29 - May 3, 1995.

[14] "International Organization for Standardization Coding of Moving Pictures and Associated Audio", ISO/IEC JTC1/SC29/WG1 1/N702, March 25, 1994 